\title{
Jugular Foramen Tumors
}

This issue has been devoted to the management of jugular foramen tumors and has been commissioned by our guest editor, Mislav Gjuric. Tumors that arise in or around the jugular foramen like paragangliomas, schwannomas, and meningiomas have always caused both surgeons and their patients very significant problems. Their resection is certainly not for the occasional skull base surgeon or indeed the faint hearted. It demands the ultimate attention to detail and, like much of what we do, the very highest levels of competence and skill.

Recent data on the natural history of many of these lesions suggests that their growth rate can be extremely slow and therefore a conservative approach can be adopted for some. Advances in imaging make this entirely appropriate as the growth rate can be monitored accurately at intervals. At the same time, recognition of the genetic defect that gives rise to the paraganglioma syndromes has reinforced the wisdom of this approach for several patients. Through screening programs in susceptible pedigrees, paragangliomas are now being detected at a stage long before they would normally have been found in days gone by. For those unfortunate patients with multiple paragangliomas there is no room for error in management planning or its subsequent execution.

The workhorse approach to the jugular foramen developed by Ugo Fisch remains the gold standard for most large jugular foramen paragangliomas. However, smaller tumors can be removed by other techniques and there is also a role for radiotherapy in some patients. The control of the internal carotid artery has also been improved by interventional radiological techniques.

All in all, it is time to reconsider the management of jugular foramen tumors, and the papers in this issue will reignite the debate.

Michael Gleeson, M.D. ${ }^{1}$ Editor-in-Chief
${ }^{1}$ Departments of Neurotology and Neurosurgery, The National Hospital for Neurology and Neurosurgery, London, United Kingdom.

Address for correspondence and reprint requests: Professor Michael Gleeson, M.D., ENT Department, 3rd Floor Thomas Guy House, Guy's Hospital, St. Thomas Street, London SE1
9RT, United Kingdom.

Jugular Foramen Tumors; Guest Editor, Mislav Gjuric, M.D.

Skull Base 2009;19:1-1. Copyright (C) 2009 by Thieme Medical Publishers, Inc., 333 Seventh Avenue, New York, NY 10001, USA. Tel: +1(212) 584-4662.

DOI 10.1055/s-0028-1124015. ISSN 1531-5010. 